\title{
AVALIAÇÃO DE DANO NO DNA EM PORTADORES DE DOENÇA PULMONAR OBSTRUTIVA CRÔNICA PELO ENSAIO COMETA
}

\author{
Helen Tais da Rosa ${ }^{1}$ \\ Andréa Lúcia Gonçalves da Silva ${ }^{2}$ \\ Martiele Bizarro ${ }^{3}$ \\ Eduarda Bender ${ }^{4}$ \\ Paulo Ricardo da Rosa \\ Anelise Araújo ${ }^{6}$ \\ Clara Forrer Charlier \\ Lia Possuelo ${ }^{8}$ \\ Dinara Jaqueline Moura \\ Andréia Rosane de Moura Valim ${ }^{10}$ \\ João Antonio Pegas Henriques ${ }^{11}$
}

\section{RESUMO}

A Doença Pulmonar Obstrutiva Crônica- DPOC é definida como uma doença respiratória inflamatória, causada principalmente pela exposição à fumaça de cigarro, caracterizada pela progressão e incompleta reversibilidade da obstrução das vias aéreas. O objetivo desta pesquisa foi quantificar o Índice de Dano ao DNA em portadores de DPOC e controles. Estudo do tipo caso-controle avaliou 43 portadores de DPOC e 50 controles emparelhados por gênero,

1 Acadêmica do Curso de Ciências Biológicas, Departamento de Biologia e Farmácia, Bolsista (PROBIC/FAPERGS da Universidade de Santa Cruz do Sul- UNISC, Santa Cruz do Sul/RS, Brasil. [htdarosa@gmail.com]

2 Departamento de Educação Física e Saúde, Universidade de Santa Cruz do Sul- UNISC, Santa Cruz do Sul/ RS, Brasil. [andreag@unisc.br]

3 Acadêmica do Curso de Ciências Biológicas, Departamento de Biologia e Farmácia, Bolsista PUIC da Universidade de Santa Cruz do Sul- UNISC, Santa Cruz do Sul/RS, Brasil. [marty_bizarro@hotmail.com]

4 Acadêmica do Curso de Ciências Biológicas, Departamento de Biologia e Farmácia, Bolsista PUIC da Universidade de Santa Cruz do Sul- UNISC, Santa Cruz do Sul/RS, Brasil. [bender.ep@gmail.com]

5 Acadêmico do Curso de Ciências Biológicas, Departamento de Biologia e Farmácia, Universidade de Santa Cruz do Sul- UNISC, Santa Cruz do Sul/RS, Brasil. [paulorlee@hotmail..com]

6 Acadêmica do Curso de Farmácia, Departamento de Biologia e Farmácia, Bolsista PUIC da Universidade de Santa Cruz do Sul- UNISC, Santa Cruz do Sul/RS, Brasil. [anelisearaújo24@yahoo.com.br]

7 Programa de Pós Graduação em Biologia Celular e Molecular da Universidade Federal do Rio Grande do SulUFRGS, Porto Alegre/ RS, Brasil. [clara.forrer.charlier@gmail.com]

8 Departamento de Biologia e Farmácia, Universidade de Santa Cruz do Sul- UNISC, Santa Cruz do Sul/ RS, Brasil. [liapossuelo@unisc.br]

9 Departamento de Biologia e Farmácia, Universidade de Santa Cruz do Sul- UNISC, Santa Cruz do Sul/ RS, Brasil e Departamento de Biofísica da Universidade Federal do Rio Grande do Sul- UFRGS, Porto Alegre/ RS, Brasil. [dinaramoura@unisc.br].

10 Departamento de Biologia e Farmácia, Universidade de Santa Cruz do Sul- UNISC, Santa Cruz do Sul/ RS, Brasil. [avalim@unisc.br]

11 Departamento de Biofísica, Universidade Federal do Rio Grande do Sul- UFRGS, Porto Alegre/RS, Brasil. [pegas@cbiot.ufrgs.br]. 
idade e índice de massa corporal (IMC). Foi utilizado sangue periférico para realizar o ensaio cometa nas versões alcalino $(\mathrm{pH}>13)$ e neutro $(\mathrm{pH} \sim 8,5)$. Avaliação de danos no DNA usando o ensaio cometa na versão alcalina revelou um maior Índice de Dano ao DNA em portadores de DPOC quando comparados aos controles (ID 40,12 $\pm 26,17$ vs ID $26,28 \pm 28,12$, $p=0,03)$. Na versão Neutra do teste também é possível observar maior Índice de Dano nos casos do que em controles (ID $52,88 \pm 32,38$ vs ID $38,12 \pm 38,17, p=0,018$ ), indicando a presença de quebras duplas, devido a inflamação persistente. Dados preliminares mostram que os portadores de DPOC têm maior índice de dano no DNA do que controles, reforçando assim a necessidade de compreender melhor os mecanismos pelos quais isso acontece no processo da doença.

Palavras-chave: DPOC. DNA. Ensaio Cometa.

\section{ABSTRACT}

Chronic Obstructive Pulmonary Disease (COPD) is defined as an inflammatory respiratory disease, mainly caused by exposure to cigarette smoke, characterized by the incomplete progression and reversibility of airway obstruction. The objective of this research was to quantify the DNA Damage Index in patients with COPD and controls. Study of case-control evaluated 43 patients with COPD and 50 controls matched by gender, age and body mass index (BMI). Peripheral blood was used to perform the comet assay versions alkaline $(\mathrm{pH}>$ 13) and neutral ( $\mathrm{pH} \simeq$ 8.5). Assessment of DNA damage using the comet assay in alkaline version showed a higher index of DNA damage in COPD patients compared to controls (ID 40.12 ID $26.28+26.17$ vs $+28.12, p=0,03)$. In the Neutral version of the test is also possible to observe higher Damage Index in cases than in controls (ID 52.88 ID $38.12+32.38$ vs $+38.17, p=0.018$ ) indicating the presence of double breaks, due to persistent inflammation. Preliminary data show that patients with COPD have a higher rate of DNA damage than controls, thus reinforcing the need to better understand the mechanisms by which this happens in the disease process.

Keywords: COPD, DNA, Comet Assay

\section{INTRODUÇÃO}

A Doença Pulmonar Obstrutiva Crônica (DPOC) está associada com uma resposta inflamatória anormal dos pulmões a partículas nocivas ou gases. As alterações decorrentes desta inflamação são progressivas e irreversíveis, destruindo os alvéolos e diminuindo a capacidade respiratória, ocasionando colapso expiratório (GOLD, 2009; ROCHA, 2005). A origem das alterações na mecânica pulmonar é devido à obstrução brônquica, que leva a um deslocamento do ponto de igual pressão para as vias aéreas que não possuem cartilagens, favorecendo o aprisionamento de ar (OLIVEIRA et al., 2000, GOLD, 2011).

A Organização Mundial de Saúde- OMS considera a DPOC uma epidemia, prevendo a sua eclosão no ano de 2020, quando poderá então se tornar a 3a maior causa de mortalidade e a 5a doença em prevalência (COSTA, RUFINO, LAPA E SILVA, 2009). O diagnóstico funcional da DPOC é confirmado através da relação do volume expiratório forçado no primeiro segundo com a capacidade vital forçada após a inalação de broncodilatadores $\left(\mathrm{VEF}_{1} / \mathrm{CVF}<0.70\right)$. A limitação do fluxo aéreo deve ser mantida em curvas 
repetidas e o diagnóstico não depende da presença de sintomas (BITTENCOURT, 2010; GOLD, 2011).

O processo inflamatório que há na DPOC tem como causa os elementos tóxicos da fumaça do cigarro. Esses gases desencadeiam alterações estruturais que predominam nas vias aéreas menores que $2 \mathrm{~mm}$. A agressão dessas estruturas provoca um processo inflamatório que tem além da presença de macrófagos, linfócitos e neutrófilos, a participação de células estruturais (epiteliais musculares e fibroblastos). A interação entre macrófagos e linfócitos, especialmente $\mathrm{CD} 8+$, tem sido associada com a patogênese da DPOC. A liberação das células recrutadas e do estresse oxidativo leva a um desequilíbrio dos mecanismos de defesa pulmonar que inicialmente é temporário. Porém, a permanência desse desequilíbrio é uma das chaves para a fisiopatogenia da doença (COSTA, RUFINO, LAPA E SILVA, 2009). A principal causa para o desenvolvimento da DPOC é o tabagismo, porém não é a única. A poluição ambiental, a exposição a químicos, a fumaça inalada, o tabagismo passivo, as infecções virais e bacterianas, e problemas respiratórios na infância também são fatores de risco que devem ser considerados. Fatores individuais devem ser incluídos como a deficiência de alfa-1-antitripsina, a deficiência da glutationa transferase, a hiperresponsividade brônquica, a desnutrição e a prematuridade (YAKSIC et al., 2003). A fumaça do cigarro é uma mistura complexa com mais de 4700 componentes químicos, com radicais livres $(\mathrm{RL})$ e outros oxidantes presentes em altas concentrações. Entretanto, estudos têm mostrado forte evidência de associação entre o tabaco e doenças respiratórias, principalmente câncer de pulmão (CEYLAN et al., 2006; BERWICK;VINEIS, 2005).

Apesar de o tabagismo ser o maior fator de risco para o desenvolvimento da DPOC, um fato interessante é que nem todos os tabagistas desenvolvem a doença, sugerindo que fatores genéticos podem modificar o risco individual. Desta forma, é possível observar que fumantes passivos desenvolvem a doença enquanto fumantes pesados não (TORRES; GODOY, 2004). O estresse oxidativo tem participação muito importante na fisiopatogenia da DPOC. O excesso de oxidantes ou a capacidade antioxidante reduzida do organismo são os principais geradores do estresse (DAHL et al., 2008).

Estudos demonstram que as espécies reativas de oxigênio (ERO) estão envolvidas nas doenças pulmonares, dentre elas a DPOC (MATSUBARA; FERREIRA, 1997; FUCHS et al, 2010). As moléculas reativas do oxigênio podem atuar aumentando a secreção de muco e a permeabilidade da membrana capilar, levando a broncoconstrição, bem como o recrutamento neutrofílico (PETTERSEN et al, 2002). O estresse oxidativo é definido como um desequilíbrio de EROs e os antioxidantes presentes no organismo que funcionam como sistema de defesa (PAGLIUSO et al, 2006). Estes podem causar danos e até morte celular, estando envolvidas em processos de envelhecimento, câncer, lesões por radiação, danos celulares, fenômenos inflamatórios e, também, com o exercício físico (PEIXOTO, 1999; MARÇAL, 2001).

O desequilíbrio redox resulta em sérias consequências a diferentes componentes celulares, tais como peroxidação de lipídeos de membrana, oxidação de proteínas e danos no DNA, que quando não são corretamente reparados, podem iniciar e promover a carcinogênese. Além disso, tem sido demonstrado que a DPOC é um predisponente para o desenvolvimento de câncer de pulmão através de vários mecanismos incluindo estresse oxidativo e processos mediados por ele, como inflamação e alteração da integridade genômica (CEYLAN et al., 2006). Neste sentido, o objetivo deste trabalho foi avaliar e quantificar o Índice de Dano ao DNA de portadores de DPOC. 


\section{MATERIAIS E MÉTODOS}

Este foi um estudo do tipo caso-controle, sendo o grupo caso composto por indivíduos portadores de DPOC e o grupo controle por indivíduos hígidos e com função pulmonar preservada. A amostragem do grupo caso foi de conveniência, caracterizada por sujeitos portadores de DPOC, da região de Santa Cruz do Sul, do programa de Reabilitação Cardiorrespiratória e Metabólica e suas Interfaces da UNISC, que ocorre periodicamente no Hospital Santa Cruz. Os controles foram selecionados a partir dos casos, entre familiares e conhecidos, bem como grupos de terceira idade que frequentam atividades de hidroginástica na UNISC, assim como integrantes da Associação de Aposentados de Santa Cruz do Sul (APOPESC), desde que atendidos os critérios de emparelhamento definidos como idade, gênero e índice de massa corporal (IMC). O estudo foi aprovado pelo Comitê de Ética e Pesquisa da Universidade de Santa Cruz do Sul sob protocolo no 2011/08. Todos os indivíduos responderam a um Questionário de Saúde Pessoal e assinaram o Termo de Consentimento Livre e Esclarecido (TCLE) antes da entrevista. A caracterização da Função Pulmonar foi realizada conforme a American Thoracic Society-ATS (1995).

\subsection{Avaliação de danos ao DNA pelo ensaio cometa}

O ensaio cometa foi realizado nas versões alcalina e neutra como descrito por Singh e colaboradores (2000). Essa é uma técnica simples, rápida e sensível para medir quebra e reparo no DNA de células individualizadas, e tem sido usada para vários estudos para investigar o efeito de EROs no DNA (DUSINSKA; COLLINS, 2008). Uma suspensão de $100 \mu \mathrm{L}$ de agarose de baixo ponto de fusão $(0,7 \%)$ e $5 \mu \mathrm{L}$ de sangue foi colocada em lâminas previamente pré cobertas com agarose $(1,5 \%)$. Uma lamínula foi colocada na lâmina e acondicionada a $4^{\circ} \mathrm{C}$ por 10 minutos, para que a agarose solidificasse. Depois disso, a lamínula foi gentilmente retirada, e a lâmina colocada em solução de lise ( $\mathrm{NaCl} 2,5 \mathrm{M}$, EDTA $100 \mathrm{mM}, 10 \mathrm{mM}$ Tris, pH 10,2, para a qual 1\% Triton X-100 e 10\% DMSO). Depois de 24h em $4 \circ \mathrm{C}$, as lâminas foram gentilmente retiradas da solução de lise. Após a lise as lâminas foram submetidas a uma corrente elétrica em cubas de eletroforese com tampão alcalino e neutro de eletroforese gelado ( $\mathrm{NaOH} 300 \mathrm{mM}$, EDTA $1 \mathrm{mM}, \mathrm{pH}>13$ e Acetato de Sódio 3M, Tris $1 \mathrm{M}$, $\mathrm{pH} 8,5$ ).

As lâminas ficaram submersas no tampão por 20 minutos (alcalino) e 1hora (neutro), para que ocorresse o desenovelamento do DNA. Após deu-se início aos procedimentos de eletroforese. Sendo as condições 25V, 300mA-0.95V/cm por 15 minutos (alcalino) e 14V, 12 $\mathrm{mA}$ por 1 hora (neutro). Após a eletroforese, as lâminas foram delicadamente retiradas da cuba e colocadas em bandejas onde foi e adicionado o tampão de neutralização (0.4MTris, $\mathrm{pH} 7,5)$. Após secas as lâminas foram fixadas e coradas com nitrato de prata de acordo com Nadin e colaboradores (2001). Para avaliação de danos no DNA, 100 células por indivíduo foram analisadas ao microscópio óptico de ampliação em 200x. Os nucleóides foram classificados visualmente de acordo com a intensidade da cauda.

\subsection{Análise Estatística}

Devido à exclusão da distribuição normal para alguns dados, as análises nãoparamétricas foram usadas para testar diferenças entre os grupos (Mann-Whitney test). As 
análises estatísticas foram realizadas utilizando o pacote estatístico SPSS 17.0 e $p \leq 0,05$ foi considerado estatisticamente significante.

\section{RESULTADOS E DISCUSSÃO}

No presente trabalho, 93 sujeitos participaram do estudo: 43 sujeitos portadores de DPOC, de ambos os sexos e 50 sujeitos controles. Os dados clínicos da amostragem revelaram o correto emparelhamento entre casos e controles, atendendo os critérios de inclusão, bem como os resultados destas variáveis vêm ao encontro da literatura (TORRES; GODOY, 2004; ALMEIDA, 2001; BRACKE et al., 2006). Nas tabelas 1 e 2 estão descritas as características clinicas e a função pulmonar dos grupos estudados.

Tabela 1 - Características Clínicas e História Tabágica dos Sujeitos.

\begin{tabular}{ccc}
\hline Características & DPOC $(\mathbf{n = 4 3})$ & Controles $(\mathbf{n}=\mathbf{5 0})$ \\
\hline Idade (anos) & 65,79 & $63,76 \pm 9,42$ \\
Sexo (M/F) & $26 / 17$ & $27 / 23$ \\
Cor da pele (branca/ não & $38 / 5$ & $49 / 1$ \\
branca) & & \\
IMC (kg/m2) & $25,17 \pm 5,95$ & $26,87 \pm 3,90$ \\
Status Tabagismo\# & $10 / 6 / 27$ & $4 / 21 / 25$ \\
DuraçãoTabagismo(>30 & 34 & 18 \\
anos) & $4(1-29)$ & $10(1-41)$ \\
Cessação Tabagismo (anos) & $28 / 8 / 3$ & $29 / 0 / 0$ \\
Tipo de Cigarro (com filtro/ & \multicolumn{2}{c}{} \\
sem filtro/ outros) & 7300 (2190-21900) & 7300 (2190-14600) \\
Cigarros/ano (ex-fumantes) &
\end{tabular}

Embora a DPOC tenha sido considerada por muito tempo uma doença de homens caucasianos velhos, dados recentes mostram que nos EUA a mortalidade está subindo muito rapidamente entre as mulheres e os africano-americanos. Os caucasianos têm apresentado menor perda de função pulmonar, por maço fumado/ ano, sugerindo que as mulheres afroamericanas são realmente mais suscetíveis aos efeitos nocivos do fumo em comparação a outros grupos de homens e mulheres. Bakos e colaboradores (2009) sugeriram que a ascendência européia como a italiana e a alemã, como é o caso deste estudo, parece ser um fator de risco a doenças pulmonares e melanomas na população da região sul do Brasil.

Embora qualquer paciente acima de 40 anos corra o risco de desenvolver esta doença, os pacientes com idade avançada têm maior risco de complicações e aumento da morbidade. Além disso, a DPOC em pessoas idosas está associada com o estado de saúde deficiente, que não pode ser previsto pelo teste de função pulmonar (CAZZOLAA; DONNERB; HANANIAC, 2008).

A carga tabágica mostrou-se superior nos portadores de DPOC quando comparados aos controles. Diversos autores propõem a existência de uma relação entre o tempo de tabagismo e a função pulmonar, mesmo esta não sendo obrigatória (TACK, 2009). 
Tabela 2 - Função Pulmonar e Histórico Breve dos Portadores de DPOC e Controles.

\begin{tabular}{lcc}
\hline & DPOC $(\mathbf{n}=\mathbf{4 3})$ & Controles $(\mathbf{n}=\mathbf{5 0})$ \\
\hline VEF $_{1}$ (\% predito) & $46,47 \pm 19,73$ & $85,98 \pm 11,78$ \\
CVF (\% predito) & $63,14 \pm 19,07$ & $90,35 \pm 12,54^{*}$ \\
VEF $_{1} /$ CVF(\% predito) & $67,93 \pm 20,40$ & $105,44 \pm 70,98^{*}$ \\
DPOC Estadiamento & $8 / 12 / 13 / 10$ & - \\
Duração da Doença (anos) & $5,97(1-43)$ & - \\
Histórico Familiar & $14 / 26 / 6$ & $10 / 33 / 7$ \\
(sim/não/ignorou) & $10 / 33$ & $10 / 40$ \\
Doença Respiratória na Infância & & \\
(sim/não) & $29 / 14$ & $37 / 13$ \\
Comorbidades (sim/não) & 23 & 27 \\
\multicolumn{1}{c}{ Cardiopatias $\quad$ Diabetes } & 9 & 2 \\
\hline
\end{tabular}

Dados apresentados em media_desvio padrão e mediana (mínimo-máximo); $\mathrm{VEF}_{1}$, volume expiratório forçado no primeiro segundo; CVF, capacidade vital forçada; ${ }^{*} p<0,05$ (Mann- Whitney Test); \#Leve/Moderado/ Severo/ Muito Severo (GOLD, 2009); HAS, hipertensão arterial sistêmica.

A função pulmonar dos casos mostrou-se reduzida quando comparada aos controles, conforme previsto, e caracterizando um quadro de obstrução pulmonar. De acordo com o GOLD (2011), o estadiamento para os portadores de DPOC deste estudo variou entre Estádio Il e V (Índice de Tiffenau < 70\%; VEF $_{1} \geq 50 \%<80 \%$ ) considerado doença moderada, severa e muito severa. Durante o segundo (II) estádio da DPOC, doença moderada, a limitação do fluxo aéreo piora, com falta de ar ao esforço, tosse e expectoração (GOLD, 2011). É nessa fase que a maioria dos doentes procura o médico, devido ao agravamento da doença e dos sintomas respiratórios crônicos. O Consenso Brasileiro sobre DPOC alerta que, nesta fase, a redução da capacidade física e a dispnéia são muito atribuídas ao envelhecimento ou ao hábito sedentário, dificultando o diagnóstico da doença precocemente (OLIVEIRA, 2000; GOLD, 2011).

Ambos os grupos apresentaram comorbidades entre elas as mais prevalentes foram a hipertensão arterial sistêmica (HAS), as cardiopatias e diabetes. A doença cardiovascular (DCV) e a DPOC também co-existem, sendo que a DCV pode estar presente em até $69 \%$ dos pacientes com DPOC e bem associada com aumento mortalidade (TACK, 2009). A fibrilação atrial também é prevalente na DPOC sendo que o tratamento com Varfarina reduz a mortalidade. Dados de um estudo longitudinal mostram que a ligação entre DPOC e a doença cardiovascular pode ser devido à inflamação local e sistêmica. Outra morbidade comum em pacientes com DPOC é a HAS por isso é necessário garantir que os pacientes em risco sejam identificados precocemente e comecem sua profilaxia (TACK, 2009).

Os resultados referentes ao EC Alcalino (Figura 1) revelaram que portadores de DPOC apresentam dano no DNA significativamente maior, quando comparados aos controles, e estes resultados estão de acordo com a literatura. No estudo realizado por Ceylan e colaboradores (2006), o Índice de Dano foi significativamente mais alto nos portadores de DPOC de origem tabágica, em relação ao encontrado nos sujeitos controles. Da mesma forma, o trabalho realizado por Maluf e colaboradores (2007), também encontrou um maior 
índice de dano em casos de DPOC quando comparados. Em nosso estudo, observamos valores de índice de dano diferente dos demais estudos, fato este que pode ocorrer devido às condições laboratoriais e sensibilidade do ensaio, pois é extremamente sensível. Referente ao grande desvio padrão no índice de dano no DNA dos nossos resultados, a heterogeneidade da amostragem, a faixa etária e as comorbidades presentes podem ter inferido sobre estes dados em ambos os grupos.

Figura 1 - Ensaio Cometa Versão alcalina (pH 10,0). Resultados do índice de danos no DNA entre casos e controles, apresentados em mediana (mínimo - máximo). Análise estatística foi realizada por Mann-Whitney Teste.

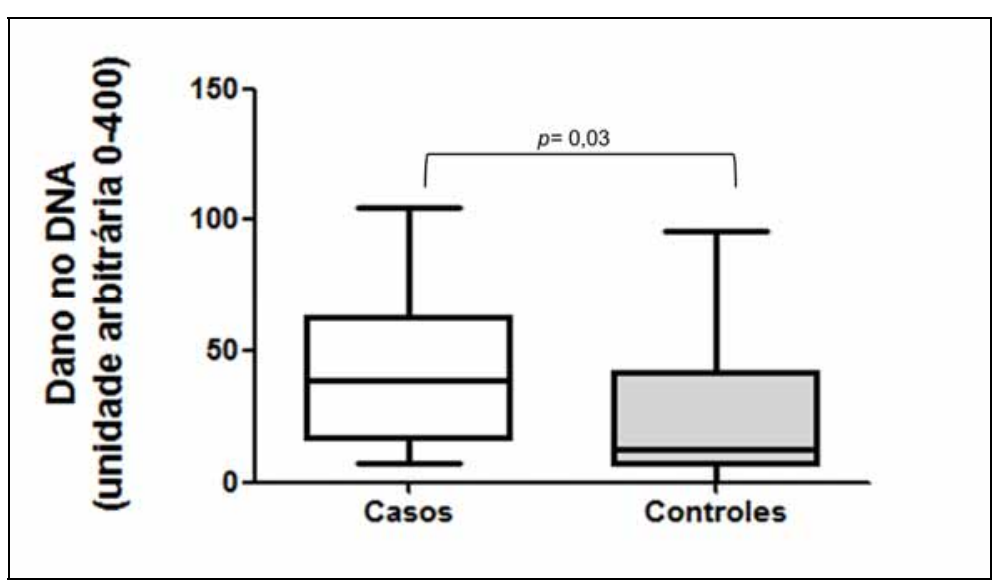

A frequência de danos e as mutações no DNA podem ser relacionadas com o hábito de fumar, pois o cigarro contém componentes químicos que contribuem para a citotoxidade e a carcinogênese. Estudos realizados com células pulmonares fetais expostas à fumaça de cigarro mostram, pelo teste cometa, que quanto maior a concentração da fumaça de cigarro, maior o dano nas células (KIM et al., 2004).

Há vários fatores que podem ser relacionados ao dano no DNA, como o metabolismo anormal de células. Cerca de $2 \%$ a $5 \%$ do oxigênio usado para a formação de ATP transforma-se em radicais livres ( $R L$ ), que podem causar danos em biomoléculas (AUGUSTO, 2006). Diferentes componentes da fumaça de cigarro reagem e podem formar diretamente $\mathrm{RL}$, enquanto as substâncias procarcinogênicas devem ser ativadas por um ou mais citocromos P450, produzindo quebras simples no DNA (REPINE et al., 1997). Modificações químicas ocorrem em todas as quatro bases do DNA, decorrentes da produção de oxiradicais, que podem quebrar o DNA e causar mutações e alterações carninogênicas (REPINE; BAST; LANKHORST, 1997). As quebras do tipo simples no DNA são os danos mais frequentes causados por EROs, que podem causar morte celular e a instabilidade genômica porém, o acúmulo de dano oxidativo e quebras do tipo simples no DNA podem ser a maior causa para a produção de quebras na dupla fita de DNA ( LAN et al., 2004).

Também observamos um maior índice de dano do tipo lesões em quebras duplas (Figura 2), avaliada pelo ensaio cometa versão neutra, o que pode ser esperado na DPOC pela presença de inflamação persistente, conforme já descrito por Repine e colaboradores (2007). 
Figura 2 - Ensaio Cometa Versão Neutra (pH 8,5). Resultados do índice de danos no DNA entre casos e controles, apresentados em mediana (mínimo-máximo). Análise estatística foi realizada por Mann-Whitney Test.

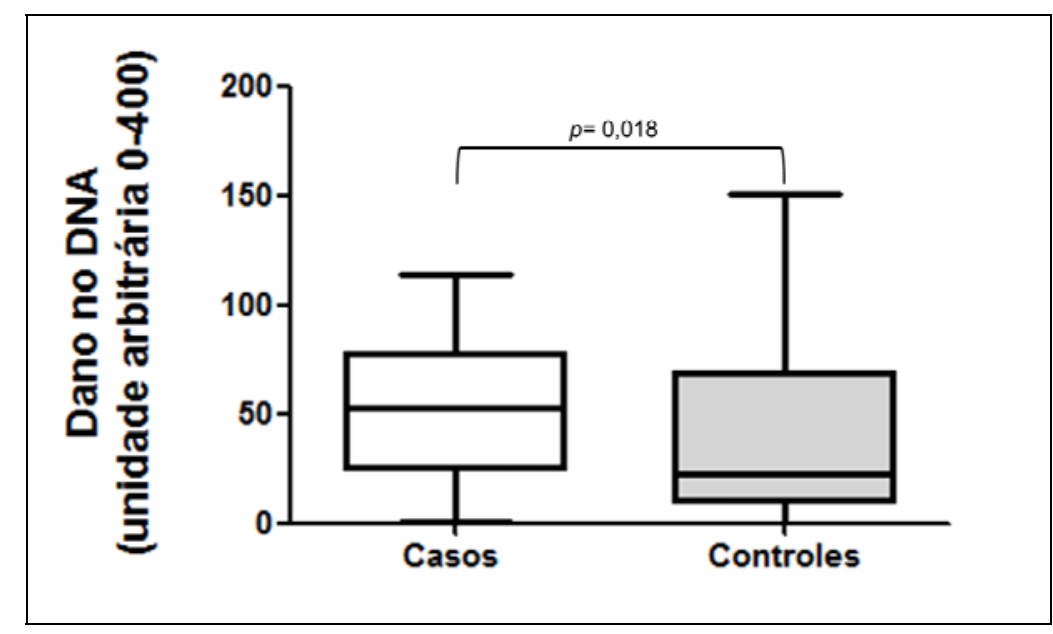

A resposta das células a danos no DNA e sua capacidade de manutenção da estabilidade genômica de reparo de DNA são a chave molecular eventos de condução de iniciação e progressão do câncer de pulmão e outras doenças pulmonares, como a DPOC (TAKABATAKE et al., 2009). O estudo de Kiechle e Zhang (2002), realizado com portadores de câncer de pulmão, revelou que esta população tem uma elevada reparação no DNA o que diminui a morte celular por apoptose em células cancerosas. Esta relação entre reparo no DNA e apoptose mostrou que a eficiência elevada de reparo no DNA pode ser considerada um dos principais obstáculos da terapia anticâncer, com base na resistência do câncer a quimioterapia. É evidente que a variação individual na capacidade de limitar os danos de DNA de carcinógenos, exógenos e endógenos contribui para o risco de diversas doenças relacionadas ao estresse oxidativo (TAKABATAKE et al., 2009).

Segundo Takabatake e colaboradores (2009), há evidências que distúrbio no balanço entre apoptose e regeneração de células estruturais pulmonares contribui para patogênese da DPOC. O incremento da apoptose em portadores de DPOC persiste mesmo após a cessação do tabagismo ocasionando um rápido declínio da função pulmonar, principalmente em pacientes mais velhos com DPOC em estágio avançado. Sendo assim, o potencial individual de suscetibilidade para o rápido declínio da função pulmonar em portadores de DPOC pode ser atribuído às variâncias genéticas que podem inferir na reparação do DNA.

\section{CONCLUSÃO}

Os dados analisados até o momento revelam um incremento no índice de dano no DNA de portadores de DPOC, quando comparados a controles sem doença pulmonar, reforçando assim a necessidade de compreender os mecanismos pelos quais isso acontece no processo da doença. Este trabalho enfatiza a importancia do Ensaio Cometa para avaliação de danos no DNA de portadores de DPOC, bem como outras doenças relacionadas ao estresse oxidativo. 


\section{REFERÊNCIAS}

ALMEIDA, A. P. B. Inflamation and chronic obstructive pulmonary disease: review article. Rev Port Pneumol, v.7, p.57, 2001.

AUGUSTO, O. Radicais Livres: Bons, maus e naturais. São Paulo: Oficina de Textos, 2006.

BERWICK M, VINEIS P. Markers of DNA repair and susceptibility to cancer in humans: an epidemiological review. J Nat Cancer Inst . v.92, p.874-97, 2000.

BRACKE, K. R. et al. Cigarette Smoke-Induced Pulmonary Inflammation and Emphysema Are Attenuated in CCR6-Deficient Mice. The Journal of Immunology, v.177, p. 4350-4359, 2006.

CAZZOLAA, M.; DONNERB,C.; HANANIAC, N. A. One hundred years of respiratory medicine chronic obstructive pulmonary disease -COPD-Republished UPDATE 4. Respiratory Medicine, p. 8-25, 2008.

CEYLAN, E. et al. Increased DNA damage in patients with chronic obstructive pulmonary disease who had once smoked or been exposed to biomass. Respiratory Medicine, v. 100, n. 1, p. 1270-1276, 2006.

COLLINS AR, HARRINGTON V. Repair of oxidative DNA damage: assessing its contribution to cancer prevention. Mutagenesis, v.17, p. 489-93, 2002.

COSTA, C.H.; RUFINO, R.; LAPA E SILVA J. R.. Células inflamatórias e seus mediadores na patogênese da DPOC. Rev. Assoc. Med. Bras. v.55, p.347-54, 2009.

DAHL, M. et al. Superoxide Dismutase 3 Polymorphism Associated with Reduced.

DUSINSKA, M. and COLLINS, A. The comet assay in human biomonitoring: geneenvironment interactions. Mutagenesis vol. 23 no. 3 pp. 191-205, 2008.

DUSINSKA, M., COLLINS, A. The comet assay in human biomonitoring: gene-environment interactions. Mutagenesis, v.23, p. 191-205, 2008.

FERREIRA, A. L. A.; MATSUBARA L. S. Radicais livres: conceitos, doenças relacionadas, sistemas de defesa e estresse oxidativo. Revista da Associação Médica Brasileira, v. 43, n. 1, p. 61-68, 1997.

FUCHS, B., et al. Redox signaling and reactive oxygen species in hypoxic pulmonary vasoconstriction. Journal Respiratory Physiology \& Neurobiology, v.174, p.282-291, 2010

Global Strategy for the Diagnosis, Management and Prevention of COPD. Iniciativa Global para a Doença Pulmonar Obstrutiva Crônica (GOLD) 2009.

Global Strategy for the Diagnosis, Management and Prevention of COPD. Iniciativa Global para a Doença Pulmonar Obstrutiva Crônica (GOLD) 2011.

KIECHLE, F.; ZHANG, X. Apoptosis: biochemical aspects and clinical implications. Clinica Chimica Acta,v. 326, p. 27-45, 2002.

$\mathrm{KIM}, \mathrm{H}$. et al. Reversible Cigarette Smoke Extract-Induced DNA Damage in Human Lung Fibroblasts. American Journal of Respiratory Cell and Molecular Biology, v. 31, n. 1, p. 483490, 2004. 
LAN, L. et al. In situ Analysis of Repair Processes for Oxidative DNA Damage in Mammalian Cells. Proceedings of the National Academy of Sciences of the United States of America, v. 101, n. 38, p. 13738-13743, 2004.

MALUF, S. W. et al. DNA damage in peripheral blood of patients with chronic obstructive pulmonary disease (COPD). Mutation Research v.626, p.180-184, 2007.

MARÇAL, L. E. Estudo do sistema NADPH oxidase e atividade glutationa peroxidase celular em granulócitos e células mononucleares de crianças e adolescentes com asma segundo a gravidade da doença. 2001, 101 f. Dissertação (Programa de Mestrado em Pediatria) Faculdade de Ciências Medicas da Universidade Estadual de Campinas, Campinas, 2001.

NADIN S. B.; VARGAS-ROIG L. M.; CIOCCA D. R. A silver staining method for single-cell gel assay. The Journal of Histochemistry \& Cytochemistry, v. 49, n. 9, p. 1183-1186, 2001.

OLIVEIRA, J. C. A., et al. I Consenso Brasileiro de Doença Pulmonar Obstrutiva Crônica (DPOC). Jornal de Pneumologia; 2000.

PAGLIUSO, R.G. et al. Estresse oxidativo e disfuncao cronica do enxerto renal. Ciencia e Saude.v. 4, p.223-227,2006. pathogenesis of lung diseases. J. Allergy Clin. Immunol.; 2008.

PEIXOTO, F. J. G. Determinação do limiar metabólico individual de lactato e estudo do estresse oxidativo em eqüinos de enduro. 1999. 101 f. Dissertação (Programa de Doutorado em Biologia Molecular e Funcional, na área da Bioquímica) - Universidade Estadual de Campinas, Campinas, 1999.

PETERSEN et al. Elevated Levels of IL-18 in Plasma and Skeletal Muscle in Chronic Obstructive Pulmonary Disease. Journal Lung, v. 185, p. 161-171, 2007.

REPINE, J. E.; BAST, A.; LANKHORST, I. and The Oxidative Stress Study Group. Oxidative Stress in Chronic Obstructive Pulmonary Disease. State of the Art. Am J Respir Crit Care Med. v. 156, p. 341-357, 1997.

ROCHA, P. F. S. Doença Pulmonar Obstrutiva Crônica (DPOC). In: Seminário apresentado ao curso de especialização em fisioterapia respiratória em UTI e ventilação mecânica com ênfase em clínica médica. São Paulo, 2005.

SINGH NP, MCCOY T, TICE RR, SCHNEIDER EL. A simple technique for of low levels of DNA damage in individual cells. Exp Cell Res; v.175, p.184-92, 1988.

TACK, G. ATS 2009 Conference Report. Respiratory medicine: COPD update. v. 5,p. $62-66$, 2009

TAKABATAKE, N. et al. A novel polymorphism in CDC6 is associated with the decline in lung function of ex-smokers in COPD. Biochemical and Biophysical Research Communications, v.381, p.554-559, 2009.

TORRES, B. S.; GODOY, I. Doenças tabaco-relacionadas. In. Diretrizes para Cessação do Tabagismo. J Bras Pneumol; v.30, p. 21-31, 2004.

YAKSIC, M. S. et al. Perfil de uma população brasileira com doença pulmonar obstrutiva crônica grave. J Pneumol, v. 29, 2003. 


\section{Agradecimentos}

Os autores agradecem aos voluntários que participaram da Pesquisa. Este trabalho foi financiado pelas agências brasileiras Fundação de Amparo a Pesquisado Estado do Rio Grande do Sul (FAPERGS), Conselho Nacional de Desenvolvimento Científico e Tecnológico (CNPq), Universidade Federal do Rio Grande do Sul (UFRGS) e a Universidade de Santa Cruz do Sul (UNISC). 